\title{
In vitro analysis of PARP inhibitor nanoformulations
}

This article was published in the following Dove Press journal: International Journal of Nanomedicine

\author{
Paige Baldwin' \\ Shifalika Tangutoori ${ }^{1,2}$ \\ Srinivas Sridhar ${ }^{1,2}$ \\ 'Nanomedicine Science and \\ Technology Center, Northeastern \\ University, Boston, MA, USA; \\ 2Department of Radiation Oncology, \\ Dana Farber Cancer Institute, Boston, \\ MA, USA
}

Correspondence: Srinivas Sridhar Nanomedicine Science and Technology Center, Northeastern University, 360 Huntington Ave, Boston, MA 02II5, USA

Email s.sridhar@neu.edu

\begin{abstract}
PARP-1 is a DNA repair protein that plays a role in a number of repair pathways and also helps in transcriptional regulation; thus PARP inhibitors (PARPi), such as olaparib and BMN-673, act by inhibiting DNA damage repair. This leads to an accumulation of deleterious mutations leading to genetic instability as a result of a number of cell replications. Currently, olaparib is only available in an oral form and has poor bioavailability, consequently leading to poor accumulation in the tumor due to first-pass metabolism. Therefore, in the present study, an injectable nanoparticle formulation of olaparib was created that offers a delivery route in which the drug would be fully bioavailable in the vasculature, suggesting greater tumor accumulation. Our results illustrated that injectable nanoformulations of olaparib and BMN-673, a next generation PARPi, could be developed, and an efficacy test indicated that BMN-673 is a much more potent PARPi than olaparib. The success of these molecular inhibitors as a monotherapy in inhibiting colony formation suggests enhanced efficacy of these treatments in combination with other therapies, even in tumors which have developed resistance.
\end{abstract}

Keywords: PARP-1, olaparib, BMN-673, DNA repair protein, tumor accumulation

\section{Introduction}

PARP inhibitors (poly(ADP-ribose) polymerase inhibitors) such as olaparib and BMN673 are among the most potent molecular inhibitors used in clinical trials for various types of cancers including prostate. PARP-1 is a DNA repair protein which plays a role in a number of repair pathways and transcriptional regulation, thus PARP inhibitors act by inhibiting DNA damage repair. This leads to an accumulation of deleterious mutations leading to genetic instability as a function of number of cell replications.

Prostate cancer tumors targeted with androgen depletion therapy tend to upregulate the transcription of the $A R$ gene. ${ }^{1}$ The crosstalk of the upregulated pathways with other oncogenic predecessors such as the TMPRSS2:ERG fusion and PTEN loss causes a resistance to therapy. PARP-1 also plays a role in the transcription pathways, and so PARPi treatment has the potential to eliminate crosstalk between the pathways leading to resensitization to treatment. ${ }^{2,3}$

Currently, olaparib is only available in an oral form, thus resulting in poor bioavailability, and consequently poor tumor accumulation due to first-pass metabolism. Therefore, an injectable nanoparticle formulation of olaparib offers a delivery route in which the drug would be fully bioavailable in the vasculature, suggesting greater tumor accumulation. Nanoformulations of olaparib and BMN-673 were developed and characterized in regard to size, charge, and loading. The efficacy of the nanoformulations was then tested on relevant cell lines to ensure the drugs retained their activity after their formulation.

\section{Materials and methods}

The prostate cancer cell line PC3 was purchased from the American Type Culture Collection (ATCC) (Manassas, VA, USA), product number CRL-1435. 

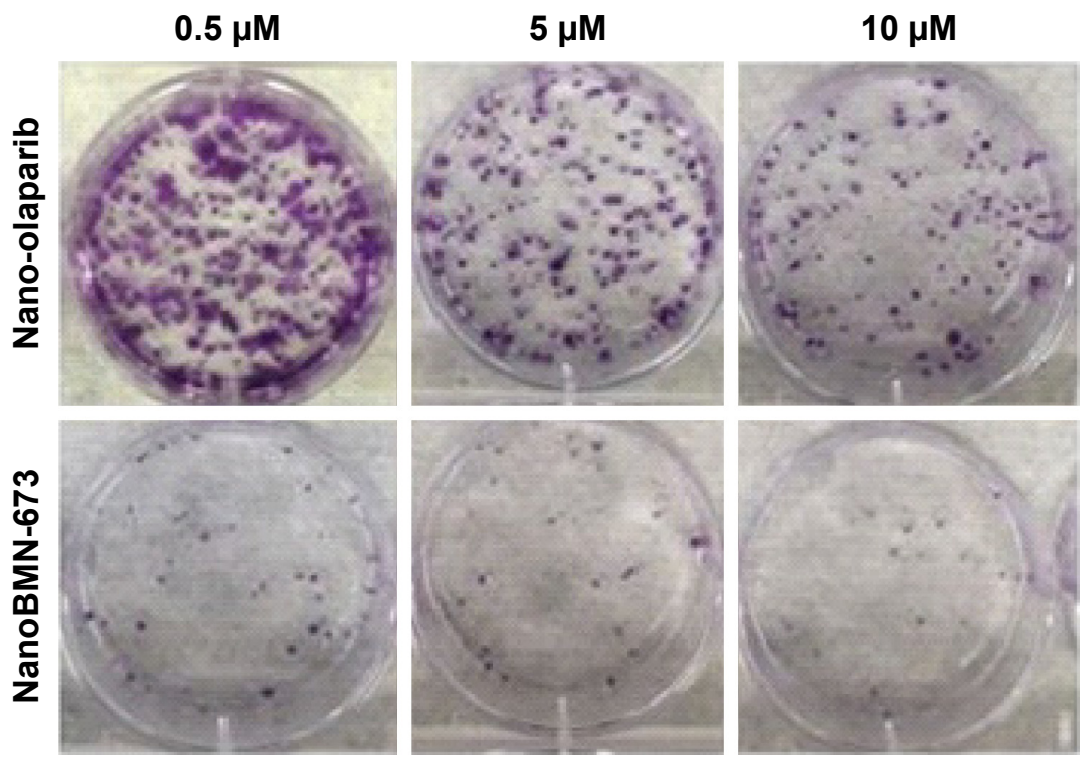

Figure I Colony formation of PC3 cells as a function of dosing for nanoformulated olaparib and BMN-673.

Abbreviation: Nano-olaparib, nanoformulated olaparib.

To compare and contrast the different nanoformulations, dose response curves were generated for each cell line to determine the half-maximal inhibitory concentrations $\left(\mathrm{IC}_{50}\right)$. Prior to this, it was crucial to develop growth rate curves for all cell lines and optimize the seeding density so as to ensure that cell death was not occurring because of overconfluence and lack of nutrients but rather due to the genetic instability caused by the treatment. These curves allow for each cell line to be exposed to the treatment of choice for 4 doubling cycles prior to analysis.

Dose response was also determined based on colony formation as shown in Figure 1. A small numbers of cells were plated after treatment with the various drug concentrations and allowed to grow in drug-free media for 10-14 days. After the allotted time frame, the cells were fixed with $10 \%$ formalin and stained with crystal violet. Colonies were counted with the stipulation that a colony is composed of at least 50 cells.

\section{Results and discussion}

$\mathrm{IC}_{50}$ for all cell lines showed BMN-673 to be a more potent PARPi than olaparib and the nanoformulations to be as potent as the free drugs (data not shown). Figure 1 pictorially illustrates this with PC3 cells (ATCC, CRL-1435), while Figure 2 quantifies the decrease in the ability of the cells to form colonies. At $0.5 \mu \mathrm{M}$, the lowest treatment dose, there is a 20 -fold decrease in colony formation between Nano-olaparib and NanoBMN-673, suggesting the next-generation PARPi BMN-673 is much more potent than olaparib.
These results are based on monotherapy treatment and indicate that, as expected, PARP-1s role in transcriptional regulation does indeed allow PARPi therapy to inhibit crosstalk between unregulated pathways. This suggests that the addition of a chemotherapeutic or radiation therapy would enhance the efficacy of either monotherapy. The efficacy of the nanoformulated drugs indicates the formulation process does not hinder drug activity and proves this is an option for enhancing the delivery of these poorly bioavailable molecular inhibitors.

\section{Conclusion}

Injectable nanoformulations of olaparib and BMN-673 have been developed to surpass the issue of bioavailability of the drugs in vivo. The efficacy of these different drugs was tested

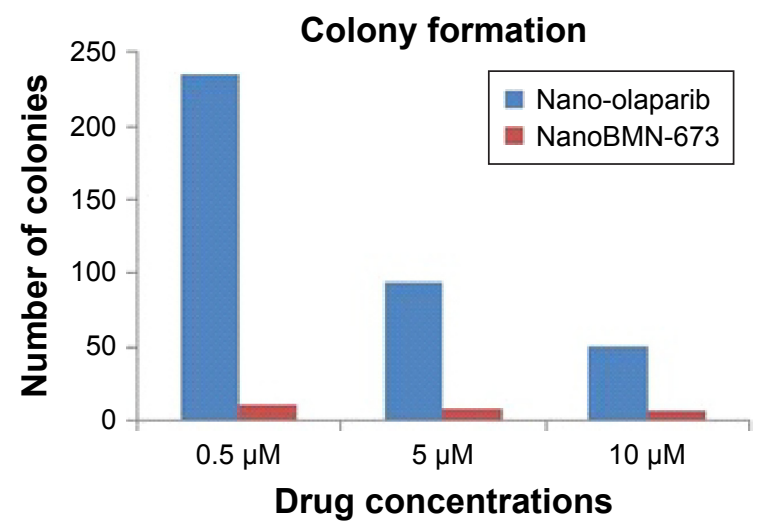

Figure 2 Quantification of the number of colonies formed at each dose. Abbreviation: Nano-olaparib, nanoformulated olaparib. 
utilizing more than one method, and the results indicate that BMN-673 is a much more potent PARPi than olaparib. The success of this molecular inhibition as a monotherapy in inhibiting colony formation suggests enhanced efficacy of these treatments in combination with other therapies, even in tumors which have developed resistance.

\section{Acknowledgment}

Supported by Integrative Graduate Education and Research Traineeship grant NSF-DGE-0965843 and Mazzone Foundation.

\section{Disclosure}

The authors report no conflicts of interest in this work.

\section{References}

1. Schiewer MJ, Goodwin JF, Han S, et al. Dual roles of PARP-1 promote cancer growth and progression. Cancer Discov. 2012;2:1134-1149.

2. Chatterjee P, Choudhary GS, Sharma A, et al. PARP inhibition sensitizes to low dose-rate radiation TMPRSS2-ERG fusion geneexpressing and PTEN-deficient prostate cancer cells. PLoS One. 2013; 8(4): 60408.

3. Brenner JC, Ateeq B, Li Y, et al. Mechanistic rationale for inhibition of poly(ADP-ribose) polymerase in ETS gene fusion-positive prostate cancer. Cancer Cell. 2011;19(5):664-678.
International Journal of Nanomedicine

\section{Publish your work in this journal}

The International Journal of Nanomedicine is an international, peerreviewed journal focusing on the application of nanotechnology in diagnostics, therapeutics, and drug delivery systems throughout the biomedical field. This journal is indexed on PubMed Central,

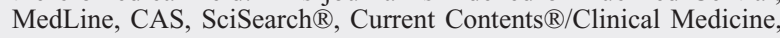

\section{Dovepress}

Journal Citation Reports/Science Edition, EMBase, Scopus and the Elsevier Bibliographic databases. The manuscript management system is completely online and includes a very quick and fair peer-review system, which is all easy to use. Visit http://www.dovepress.com/ testimonials.php to read real quotes from published authors. 